ARTICLE OPEN

\title{
A first-principle perspective on electronic nematicity in FeSe
}

\author{
Xuanyu Long ${ }^{1}$, Shunhong Zhang $\mathbb{D}^{2}$, Fa Wang ${ }^{3,4}$ and Zheng Liu $\mathbb{D}^{1,4 凶}$
}

Electronic nematicity is an important order in most iron-based superconductors, and FeSe represents a special example, in which nematicity disentangles from spin ordering. A first-principle description of this order remains elusive. Here, we show that by carefully searching the paramagnetic energy landscape within the density functional theory, a nematic solution stands out at either the $+U$ or hybrid functional level with the lowest energy. The band structure and Fermi surface can be well compared with the recent experimental results. Symmetry analysis assigns the dominant order parameter to the $E_{\mathrm{u}}$ irreducible representations of the $D_{4 \mathrm{~h}}$ point group. Distinct from the $B_{1 \mathrm{~g}}$ Ising nematicity as widely discussed in the context of vestigial stripe antiferromagnetic order, the two-component $E_{\mathrm{u}}$ vector order features mixing of the Fe $d$-orbitals and inversion symmetry breaking, which lead to striking experimental consequences, e.g., missing of an electron pocket.

npj Quantum Materials (2020)5:50; https://doi.org/10.1038/s41535-020-00253-x

\section{INTRODUCTION}

First-principle calculation within the framework of density function theory (DFT) has played an important role in understanding the iron-based superconductors. For the high-temperature paramagnetic (PM) phase, the standard local density approximation (LDA) or its generalized gradient approximation (GGA) extension can already qualitatively describe the Fermi surface topology and its orbital components $^{1-3}$. For the low-temperature magnetic phase, the local spin density approximation (LSDA) or spin-polarized GGA (sGGA) can obtain the correct ground-state spin order in most cases $^{1,2,4}$. Additional corrections to the electronic correlation effects, e.g., the dynamical mean-field theory (DMFT), further reduce the quantitative discrepancies, such as the overestimation of the band width and the magnetic moment ${ }^{5,6}$.

However, a first-principle description of the nematic order remains elusive. Dictated by a fourfold rotational $\left(C_{4}\right)$ symmetry breaking, the nematic phase remains as a PM metal ${ }^{7}$. The attempt to explicitly break the $C_{4}$ symmetry by straining the lattice in the DFT simulation results in only negligible changes on the electronic structure $^{8}$, which is not surprising, since there is strong experimental evidence suggesting that the nematic order is of an electronic origin ${ }^{9}$.

Useful insights can be gained from different perspectives ${ }^{7}$. One scenario that finds a wide range of applications in iron pnictides is to view it as a precursor of the stripe antiferromagnetic phase, in which the spin quadrupolar fluctuation first diverges before the ordering of the spins ${ }^{10-12}$. The other is to invoke a spontaneous orbital ordering ${ }^{13-15}$. For a highly correlated material, the "truth" probably lies between. Despite very different microscopic origins, by symmetry, the formulated order parameters (OPs) all belong to the one-dimensional $B_{1 \mathrm{~g}}$ irreducible representation (irrep) of the $D_{4 \mathrm{~h}}$ point group, i.e, Ising nematicity. Therefore, these OPs are intertwined $^{16}$, and in reality electronic nematicity manifests in both the spin and the orbital degrees of freedom concurrently. There is no sharp criterion for distinguishing them.

From the DFT perspective, it is difficult to take a composite spin order into account. Nevertheless, mature orbital-resolved approaches, e.g., DFT $+U^{17}$ and hybrid functional ${ }^{18}$, allow probing potential instabilities in the orbital channel. FeSe represents an ideal platform to perform this numerical experiment, because no magnetic order has thus far been observed in bulk FeSe, unless high pressure is applied ${ }^{19,20}$. This provides a chance to study nematicity disentangled from spin ordering, and it is reasonable to speculate that in this case the orbital instability, if exists, can be detected by DFT $+U$ and hybrid functional calculations. In addition, inspired by the high superconducting transition temperature of monolayer $\mathrm{FeSe}$ on $\mathrm{SrTiO}_{3}$ substrate ${ }^{21,22}$, extensive experimental data have been collected and crosschecked, in particular highresolution angle-resolved photoemission spectroscopy (ARPES) data ${ }^{8,23-38}$, and thus systematic evaluation of the calculation results is possible.

In previous theories, orbital splitting with opposite signs around $\Gamma$ and $M$ points is the dominant order parameter reproducing the ARPES observations ${ }^{39-41}$, but this OP cannot arise from mean-field treatment of local correlations on single $\mathrm{Fe}$ atoms, and sophisticated many-body theory has to be employed ${ }^{40,41}$.

Here, we show that the important features of the nematic phase can actually be captured by careful treatment of local correlations within the state-of-the-art first-principle framework. The results suggest a predominant nematic OP belonging to the twodimensional $E_{\mathrm{u}}$ irreducible representations of the $D_{4 \mathrm{~h}}$ point group. The coexistence of $C_{4}$ rotational and inversion symmetry breaking associated with the $E_{\mathrm{u}} \mathrm{OP}$ is found as the key to reproduce the experimental energy bands and Fermi surface.

\section{RESULTS AND DISCUSSION}

Nematic solutions

Figure 1(a) schematically summarizes three self-consistent solutions emerged in our DFT $+U$ calculation: $a$-solution is the symmetric one commonly obtained in previous study; $\beta$ and $\gamma$ are the two symmetry-breaking solutions not known before. Within our calculation framework, the $\gamma$-solution presents as the lowest-energy state. Figure 1(b) shows the electron-density contour of the a solution $\left[\rho_{a}(\mathbf{r})\right]$. Figure $1(\mathrm{c}, \mathrm{d})$ shows the

\footnotetext{
${ }^{1}$ Institute for Advanced Study, Tsinghua University, 100084 Beijing, China. ${ }^{2}$ International Center for Quantum Design of Functional Materials (ICQD), Hefei National Laboratory for Physical Sciences at the Microscale, University of Science and Technology of China, Hefei, 230026 Anhui, China. ${ }^{3}$ International Center for Quantum Materials, School of Physics, Peking University, 100871 Beijing, China. ${ }^{4}$ Collaborative Innovation Center of Quantum Matter, 100084 Beijing, China. ${ }^{\circledR}$ email: zheng-liu@tsinghua.edu.cn
} 


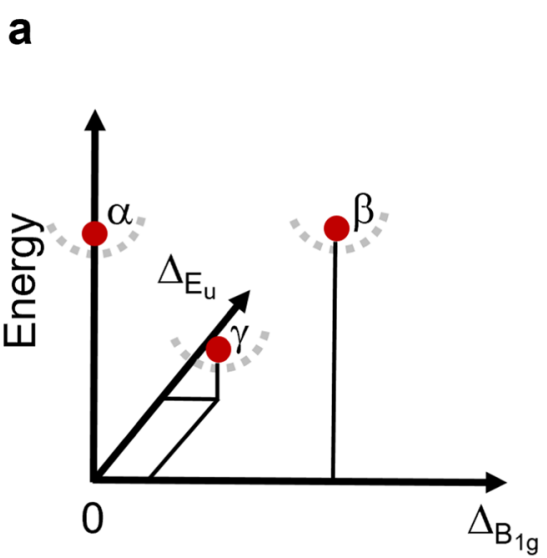

C

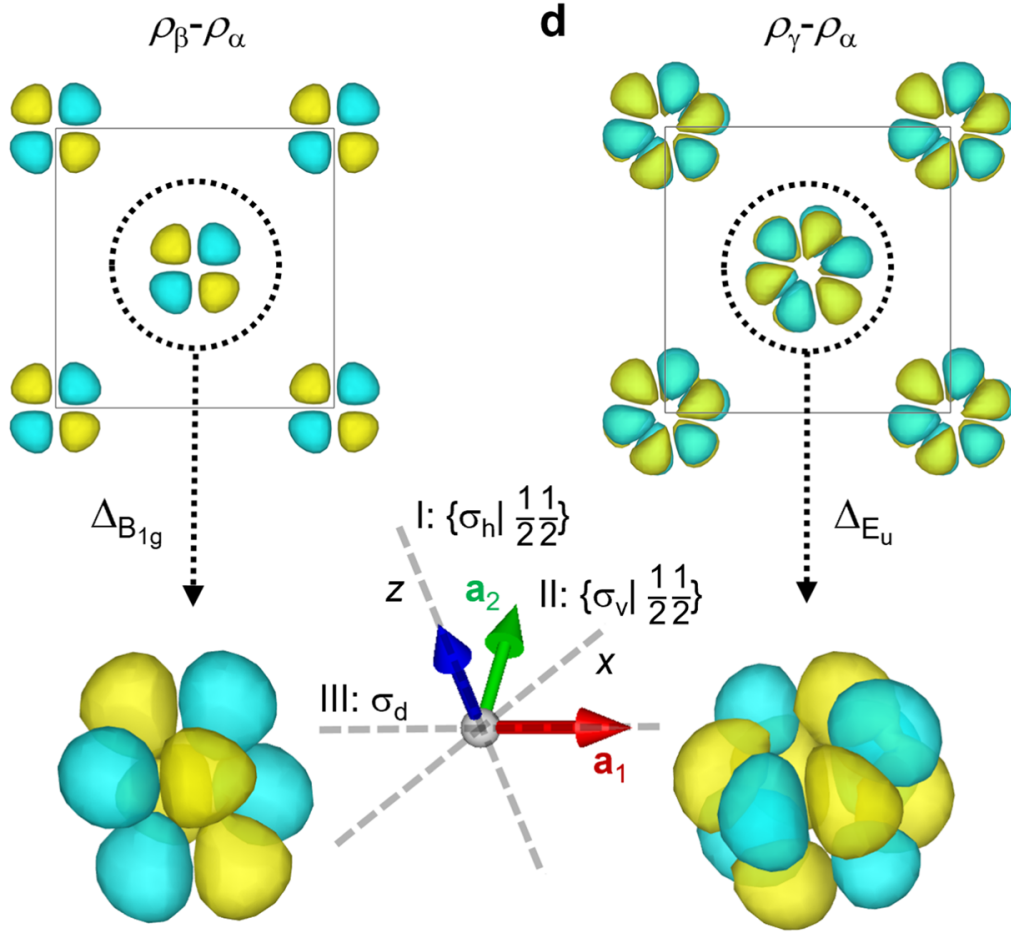

b

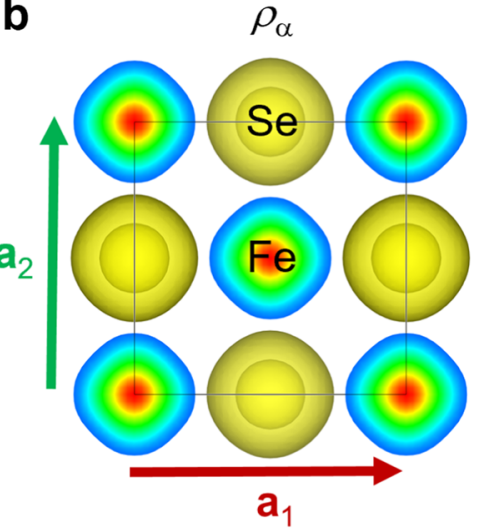

$\mathbf{a}_{1}$

(2)

Fig. 1 Self-consistent symmetric and symmetry-breaking solutions. a A schematic illustration of multiple self-consistent solutions in the energy landscape. $\mathbf{b}$ Charge density contour of the symmetric solution $\left(\rho_{a}\right)$ with $\mathbf{a}_{1,2}$ as the two lattice vectors of the 2-Fe unit cell; $\mathbf{c}$, $\mathbf{d}$ Charge density change of the two symmetry-breaking solutions $\left(\delta \rho_{\beta}\right.$ and $\left.\delta \rho_{\gamma}\right)$. The blue (yellow) color of the isovalue contour stands for the positive (negative) sign of $\delta \rho$. The zoomed-in distribution of $\delta \rho$ in three dimensions around a single Fe, i.e., the orbital-order parameters $\Delta_{B_{19}}$ and $\Delta_{E_{\mathrm{u}}}$ are displayed below. The inset shows the three generators of the $P 4 / n m m / \mathcal{T} \cong D_{4 \mathrm{~h}}$ point group. The dashed gray lines are the normals of the reflection mirrors, which pass an Fe atom.

differences between the electron-density contour of the $\beta$ and $\gamma$ solutions $\left[\rho_{\beta / \gamma}(\mathbf{r})\right]$ and $\rho_{\alpha}(\mathbf{r})$.

In Fig. 2, we reproduce the band strucuture and Fermi surface of the symmetric phase, which has been well established in the literature ${ }^{1-3}$. An impressive achievement of DFT is the capability of capturing the band connections, orbital components, and Fermi surface topology qualitatively, despite the significantly overestimated band width $^{42}$. Overall, the $D F T+U$ results of the symmetric a solution shown in Fig. 2(c, d) are not much different from the plain DFT ones [See, for example, Chpt. 8 in ref. ${ }^{1}$ ]. Turning to the nematic phase Fig. 3(a, b), a series of band reconstructions are clearly resolved in recent ARPES data ${ }^{25,27-32,37,38,43,44}$. It is striking that the lowest-energy $\gamma$ solution captures nearly all the qualitative features. Specifically, around $\Gamma$, the $d_{x z}$ and $d_{y z}$ bands are clearly split, and the hole Fermi surface becomes anisotropic. Around $M$, the bands remain largely unchanged on the $\Sigma_{x}$ side, whereas on the $\Sigma_{y}$ side, the crossing between the $d_{x z}$ and $d_{y z}$ bands is gapped out, regrouping the bands into a hybridized upper branch and a lower branch. One remarkable consequence is that the electron Fermi surface loses the lobes along the gapped direction, which is termed as the "missing electron pocket" ${ }^{137}$, as also observed in refs $25,27-32,38,43,44$.

The $\beta$ solution captures the hole band splitting, but it fails to reproduce the characteristic "one-sided" gapping. The $\beta$ bands can be largely viewed as a momentum-independent upward shift of the $d_{x z}$ bands. In contrast, the $\gamma$ bands have a more complicated structure.

We note that Fig. 3(c, d) are not fine-tuned results. In Fig. 4, we switch to a different version of the $+U$ correction, and benchmark the $\mathrm{DFT}+U$ bands to the hybrid functional results. The latter contains no material-specific parameters, and the lattice constants and atomic positions are fully relaxed. All these methods 
a

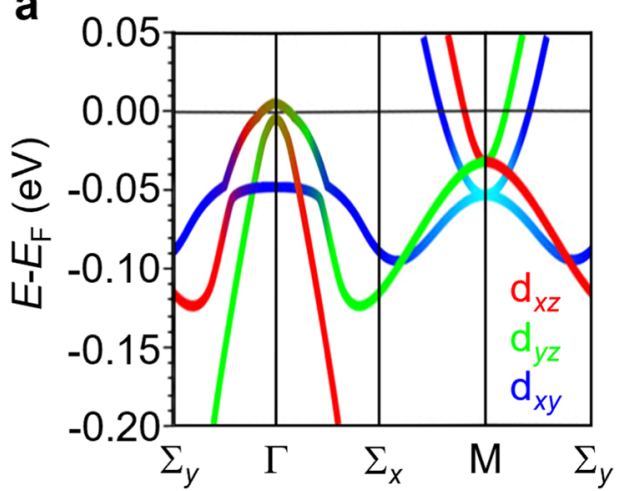

C

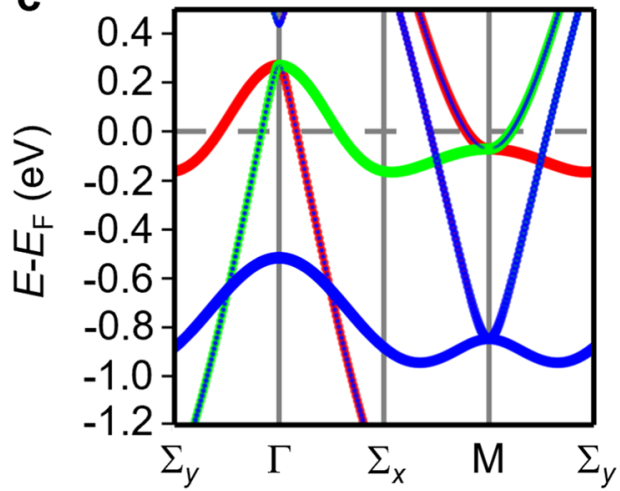

b

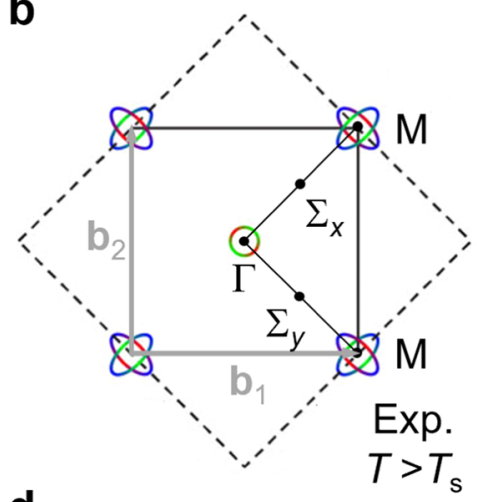

d

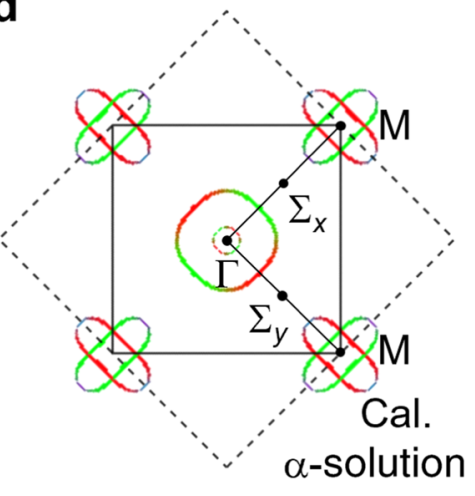

Fig. 2 Band structure and Fermi surface of the symmetric phase. The schematics of experimental results (a) and (b) are replotted based on the ARPES data in ref. ${ }^{37} ; \mathbf{c}, \mathbf{d}$ are the calculated bands and $k_{z}=0$ Fermi surface slices of the symmetric $a$ solution. The color of the bands and Fermi surfaces denotes the orbital components [inset of $\mathbf{a}$ ], following the convention of ref. ${ }^{33} . \mathbf{b}_{1}$ and $\mathbf{b}_{2}$ are the two reciprocal vectors of the 2-Fe Brillouin zone. The dashed square shows the 1-Fe Brillouin zone, in which $\Gamma-\Sigma_{x}\left(\Sigma_{y}-\Gamma\right)$ corresponds to the $x(y)$ direction.

consistently reproduce the $\gamma$ solution as the ground state and nicely reproduce the experimental nematic band structures.

Figure 5 shows how the DFT $+U$ total energy of the three solutions change when we manually change the $+U$ parameter $(U)$, Fe-Fe distance $(a / \sqrt{2})$ and the out-of-plane coordinate of Se $\left(z_{\mathrm{Se}}\right)$. The overall trend is that nematicity is favored by (a) a larger $U$ (interaction driven) and (b) larger $a / \sqrt{2}$ and $z_{\mathrm{se}}$. Compared with $a / \sqrt{2}, z_{\mathrm{Se}}$ appears as a more sensitive factor.

Figure 6 summarizes the ground-state band evolution as we change the microscopic details. It is informative to observe how the nematic band structure deforms back to the symmetric one on the left side of the figure. During the process, the $d_{x z}-d_{x y}$ gap along the $\mathrm{M}-\Sigma_{y}$ direction gradually closes. The linear crossing without hybridization forms in the end.

Key ingredients to obtain the nematic solutions

There are several important theoretical considerations underlying the calculations, which distinguish the present work, and also explain why the nematic solutions were not identified in previous DFT and DMFT calculations.

$P M$ condition. Within the nematic phase, FeSe is experimentally a PM metal. LSDA or sGGA has been shown to result in a quasidegenerate lowest-energy manifold with stripe magnetic ordering momenta $(\pi, Q)$ and $(Q, \pi)(0<Q \leq \pi / 2)^{45,46}$. The real spin state of the nematic phase has no long-range order. Based on the LSDA (sGGA) results, one natural speculation is a cooperative PM state ${ }^{45,46}$, i.e. fluctuations within the lowest-energy manifold restore the time-reversal symmetry. To comply with the $C_{4}$ rotation symmetry breaking, it additionally requires that the fluctuations spontaneously condense into either the $(\pi, Q)$ or $(Q, \pi)$ direction. Another possibility is that the LSDA (sGGA) manifold actually collapses into a quantum PM state ${ }^{47}$. In contrast to the previous spin-polarized calculations, the present work focuses on nonmagnetic calculations, without introducing any spin order. Practically, we employ GGA instead of sGGA. For a PM metal, this is considered as a reasonable starting point.

Interaction correction. We have employed either DFT $+U$ correction $^{17}$ or hybrid functional ${ }^{18}$ to upgrade the description of strong interactions associated with the Fe $d$-electrons from an orbitalindependent mean field to an orbital-dependent potential, which is the key to capture the orbital-ordering instabilities. Successful applications of these corrections to iron-based superconductors were hindered by the observation that $+U$ tends to increase the error of ordered spin moment in the magnetic phase, as already overestimated at the LSDA (sGGA) level. This problem is automatically avoided in our nonmagnetic calculation. A unified view of the $+U$ correction and the hybrid functional under the PM condition is that they both tend to cancel the unphysical Hartree potential of an electron with itself, which represents one of the most conspicuous error in LDA (GGA), and is significant for a localized orbital.

Wavefunction preconditioning. For a correlated system like FeSe, the energy landscape could be rather complicated. In consequence, numerical minimization might be easily trapped to some local minimum points ${ }^{48-50}$. In particular, starting from an initial electron-density $\rho_{0}(\mathbf{r})$ that respects the full symmetry of the underlying lattice, the iteration can easily end at a $\rho_{\mathrm{GS}}(\mathbf{r})$ without symmetry breaking. Therefore, to probe potential symmetry breaking, it is demanded to purposely drag $\rho_{0}(\mathbf{r})$ away from the symmetric basin by preconditioning the initial trial wavefunctions $\left\{\psi_{i}(\mathbf{r})\right\}$. Other preconditioning approaches, e.g., imposing an initial density matrix for correlated orbitals, can serve the same goal ${ }^{49,50}$. 

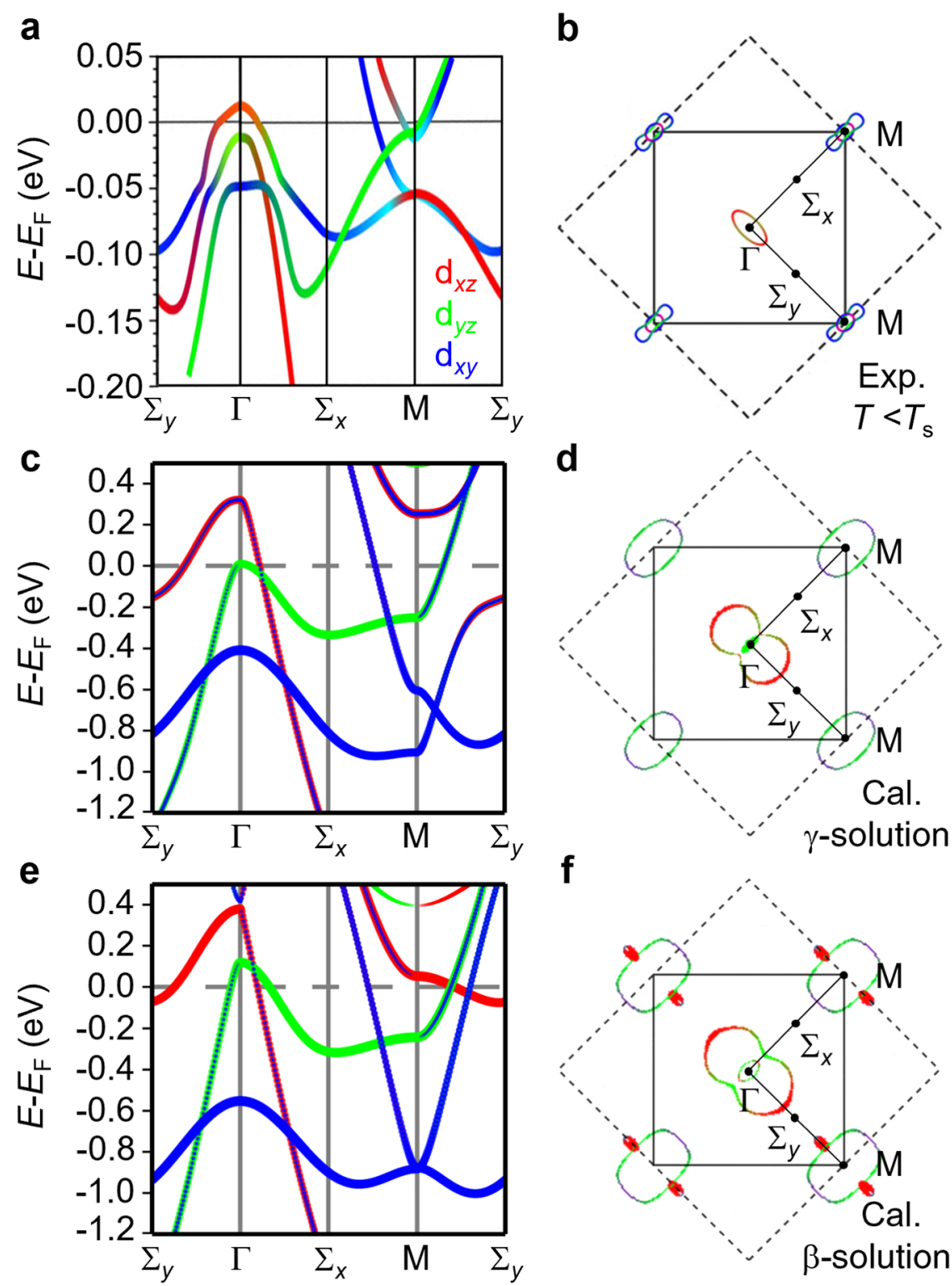

Fig. 3 Band structure and Fermi surface of the nematic phase. The schematics of experimental results (a) and (b) are replotted based on the ARPES data in ref. ${ }^{37} ; \mathbf{c}, \mathbf{d}$ and $\mathbf{e}, \mathbf{f}$ are the numerical results of the $\gamma$ and $\beta$ solutions.

OP analysis

The electron-density change $\left[\delta \rho_{\beta / \gamma}(\mathbf{r})\right]$ with respect to the symmetric solution as shown in Fig. 1(c, d) exhibits clear symmetry breaking patterns. $\delta \rho_{\beta}(\mathbf{r})$ can be easily associated with the widely discussed ferro-orbital nematic order. It is known that this OP is Ising type, belonging to the 1D $B_{1 \mathrm{~g}}$ irrep of the $D_{4 \mathrm{~h}}$ point group. By inspecting the density matrix $\tilde{n}^{\alpha \beta}$ within the Fe $3 d$-orbital subspace, where $a$ and $\beta$ label the five $3 d$-orbitals of the Fe atom, we confirm that the most noticeable change is $\tilde{n}^{y z, y z}-\tilde{n}^{x z, x z} \neq 0$. The $x, y$ axes are along the Fe-Fe bonds. Accordingly, the symmetry breaking OP can be written as:

$\Delta_{B_{19}}=\tilde{n}^{y z, y z}-\tilde{n}^{x z, x z}$.

Following the choice of the three group generators in ref. ${ }^{51}$ [See also the inset of Fig. $1(c, d)$ ], we list the transformation matrices (parities for $\Delta_{B_{19}}$ ) in Table 1.

$\delta \rho_{\gamma}(\mathbf{r})$ in the form of high-rank multipoles is however unexpected. By inspecting $\tilde{n}^{\alpha \beta}$, a fraction of $\Delta_{B_{1 g}}$ is also present in the $\gamma$ solution, but an even larger change is the emergence of nonvanishing off-diagonal terms: $\tilde{n}^{x z, x y} \simeq \tilde{n}^{y z, x^{2}-y^{2}}$ (and the complex conjugate, $\tilde{n}^{x y, x z} \simeq \tilde{n}^{x^{2}-y^{2}, y z}$ ). We define this orbital hybridization as a new OP:

$\Delta_{E_{\mathrm{u}, 1}}=\tilde{n}^{x z, x y}+\tilde{n}^{y z, x^{2}-y^{2}}$

This is not an Ising OP, which can be most clearly seen by rotating Fig. $1(\mathrm{c}, \mathrm{d})$ by $90^{\circ}$. While Fig. 1(c) simply changes the sign, Fig. $1(\mathrm{~d})$ is transformed into an inequivalent orientation. Formally, the other symmetry-related OP is obtained by interchanging $x$ and $y$ :

$\Delta_{E_{\mathrm{u}, 2}}=\tilde{n}^{y z, x y}-\tilde{n}^{x z, x^{2}-y^{2}}$

These two OPs form a 2D $E_{\mathrm{u}}$ irrep. We list in Table 1 the associated transformation matrices.

It is interesting to note that the combination of the two terms in Eqs. (2) and (3) leads to an analytically compact form of 
a

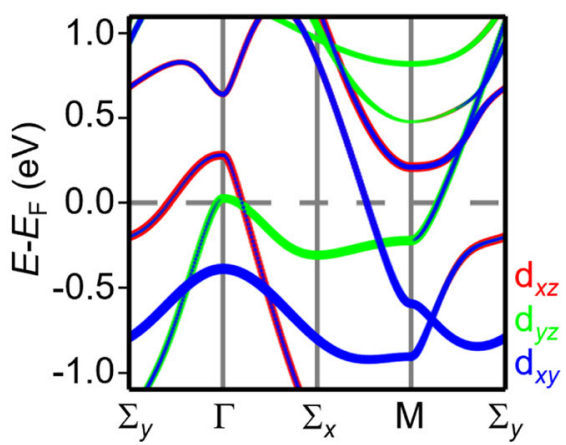

b

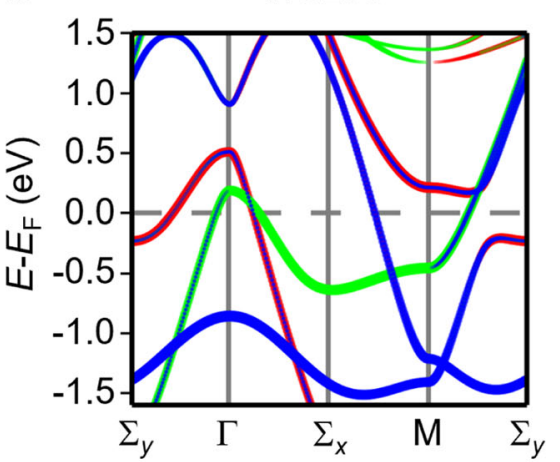

Fig. 4 The ground-state ( $\gamma$ solution) band structure calculated by two other methods. a The full orbital-dependent $+U$ correction $^{67}$; and $\mathbf{b}$ HSE06 ${ }^{61,62}$ hybrid functional.
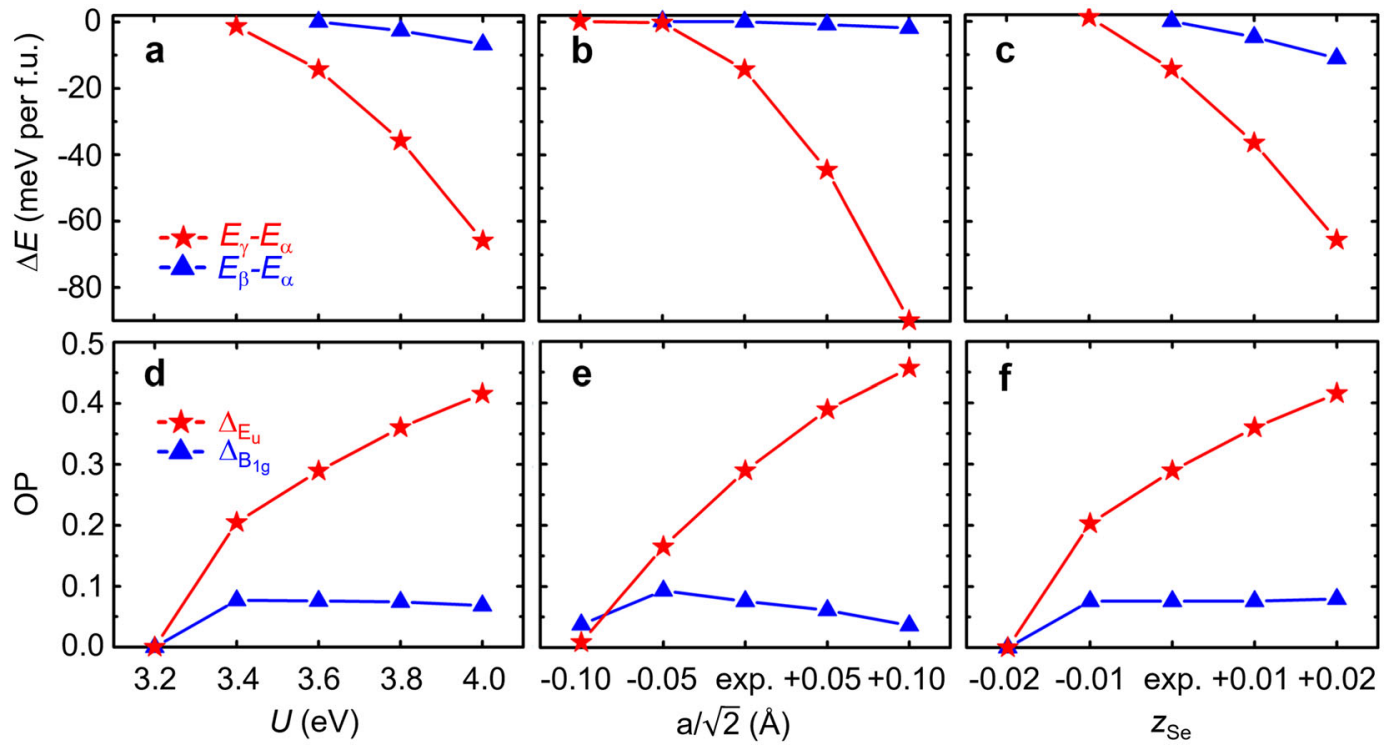

Fig. 5 The evolution of total energy and OP amplitude. a-c The total energy of the $\beta$ (blue triangles) and $\gamma$ (red stars) solutions; and $\mathbf{d}-\mathbf{f}$ the OP amplitude $\Delta_{E_{u}}$ (red stars) and $\Delta_{B_{1 g}}$ (blue triangles) of the ground state ( $\gamma$ solution) as a function of the the $+U$ parameter (U), Fe-Fe distance $(a / \sqrt{2})$ and the out-of-plane coordinate of Se $\left(z_{\mathrm{Se}}\right)$.

electron-density modulation around Fe atoms:

$$
\begin{aligned}
\delta \tilde{\rho}_{E_{\mathrm{u}, 1}}(\mathbf{r})= & \Delta_{E_{\mathrm{u}, 1}}\left[\psi_{x z}(\mathbf{r}) \psi_{x y}(\mathbf{r})+\psi_{y z}(\mathbf{r}) \psi_{x^{2}-y^{2}}(\mathbf{r})\right] \\
& \sim R_{3 d}^{2}(r) \sin ^{3} \theta \cos \theta \sin 3 \phi \\
& \sim Y_{4}^{3}(\theta, \phi)+Y_{4}^{-3}(\theta, \phi)
\end{aligned}
$$

in which $R_{3 d}(r)$ is the radial wavefunction of $3 d$-orbitals and $(\theta, \phi)$ is the angular coordination of $\mathbf{r}$. Similarly,

$\delta \tilde{\rho}_{E_{\mathrm{u}, 2}}(\mathbf{r}) \sim Y_{4}^{3}(\theta, \phi)-Y_{4}^{-3}(\theta, \phi)$.

It is straightforward to check that the analytical $\delta \tilde{\rho}_{E_{\mathrm{u}, 1}}(\mathbf{r})$ indeed reproduces the overall geometry of $\delta \rho_{\gamma}$ [Fig. 1(d)]. It can be viewed as a hexadecapolar order.

According to Fig. 5(d-f), $\Delta_{E_{u}}$ and $\Delta_{B_{1 g}}$ typically coexist in the ground state, but $\Delta_{E_{\mathrm{u}}}$ is the leading one. The existence of the $E_{\mathrm{u}}$ OPs will generically generate the $B_{1 \mathrm{~g}}$ order parameter, due to the symmetry-allowed coupling $\Delta_{B_{19}}\left(\Delta_{E_{\mathrm{u}, 1}}^{2}-\Delta_{E_{\mathrm{u}, 2}}^{2}\right)$ in an effective Ginzburg-Landau-type theory. The pure $\Delta_{B_{1 \mathrm{~g}}}$ ( $\beta$ solution) always has a higher energy.
Experimental consequences

Band reconstructions. Identification of the OPs reveal the physical origin of band reconstructions shown in Fig. 3. According to Table 1, both $\Delta_{B_{1 g}}$ and $\Delta_{E_{\mathrm{u}}}$ breaks $\left\{\sigma_{\mathrm{v}} \mid \frac{1}{2} \frac{1}{2}\right\} \times \sigma_{\mathrm{d}}$, and thus the $C_{4}$ rotation, leading to nematicity. The hole band splitting is primarily associated with this symmetry breaking, and the $\beta$ and $\gamma$ solutions behave similarly. The "hidden order" in $\Delta_{E_{u}}$ is related to the further breaking of $\left\{\sigma_{v} \mid \frac{1}{2} \frac{1}{2}\right\}$. The remaining symmetry shrinks to a $C_{2 v}$ group. We list in Table 2 the little groups of the three solutions. It is important to notice that $\Delta_{B_{1 g}}$ does not lower the symmetry of the ordinary k-points along $\Sigma_{x(y)}$ directions. However, $\Delta_{E_{\mathrm{u}}}$ lowers the symmetry along $\Sigma_{y}$ from $C_{2 v}$ to $C_{s}$. The missing group element $\left\{\sigma_{v} \mid \frac{1}{2} \frac{1}{2}\right\}$ originally protects the $d_{x z}$ and $d_{x y}$ band crossing near the $M$ point. This symmetry reason naturally explains why the "onesided" gapping occurs, without the requirement of band inversion $^{37}$ or orbital-selective quasiparticle weight ${ }^{43,44}$ as previously proposed. From a different angle, the "one-sided" gapping can be understood from the first term on the right hand side of Eq. (2). This hybridization term tends to mix $d_{x z}$ and $d_{x y}$ bands. On the other hand, $d_{y z}$ is coupled to $d_{x^{2}-y^{2}}$ only, which lies away from the Fermi surface. The crossing point between $d_{y z}$ and $d_{x y}$ bands is thus largely unperturbed. The redistribution of electron 


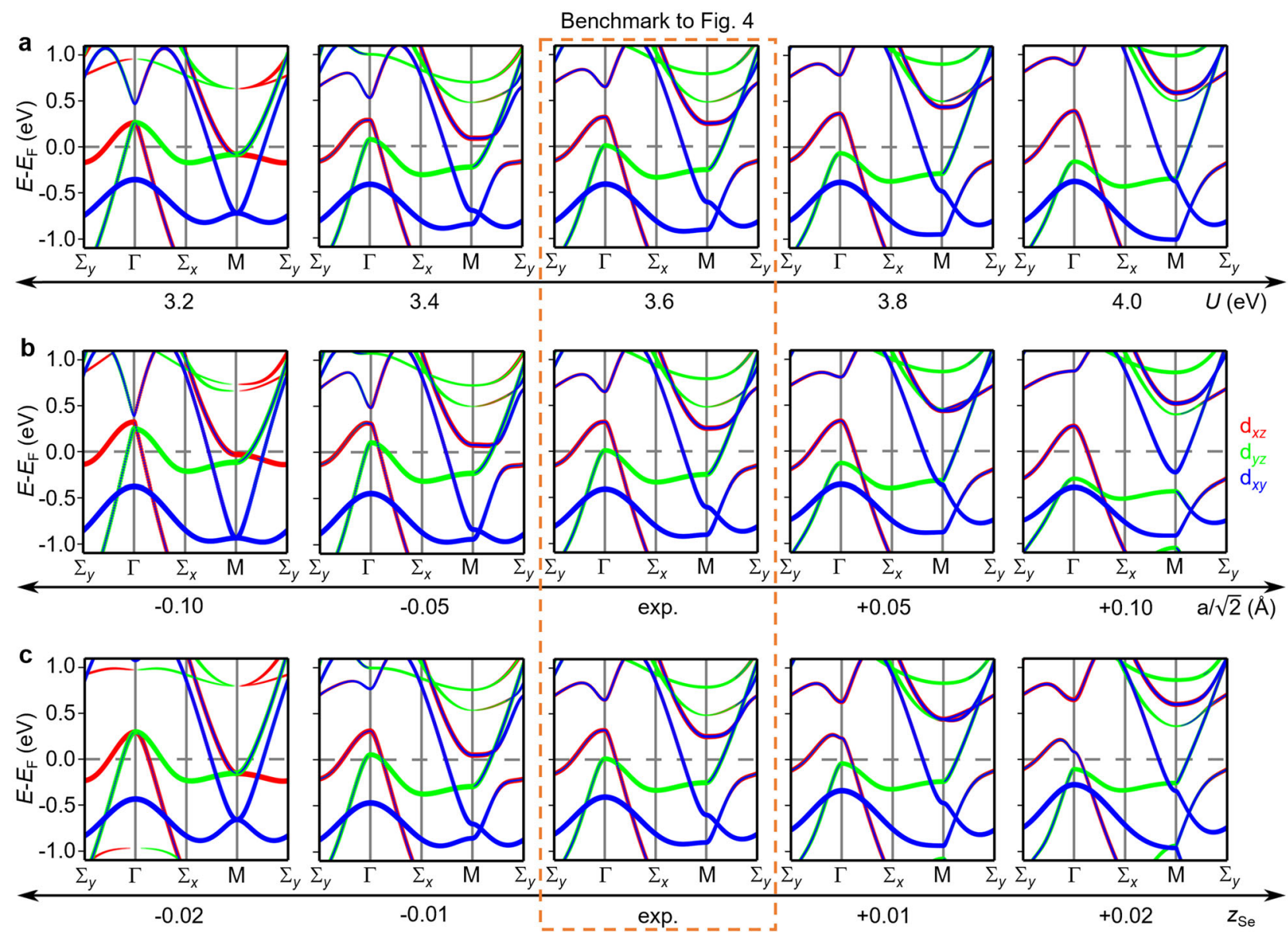

Fig. 6 Ground-state band evolution. Band structure as a function of a the $+U$ parameter $(U), \mathbf{b}$ Fe-Fe distance $(a / \sqrt{2})$ and $\mathbf{c}$ the out-of-plane coordinate of Se $\left(z_{\mathrm{Se}}\right)$.

Table 1. Transformation matrices of the predicted OPs with respect to the three generators of the $P 4 / n m m / \mathcal{T} \cong D_{4 h}$ point group.

\begin{tabular}{|c|c|c|c|}
\hline $\mathrm{OP}$ & $\left\{\sigma_{\mathrm{h}} \mid \frac{1}{2} \frac{1}{2}\right\}$ & $\left\{\sigma_{\mathrm{v}} \mid \frac{1}{2} \frac{1}{2}\right\}$ & $\sigma_{\mathrm{d}}$ \\
\hline $\begin{array}{l}\Delta_{B_{19}} \\
\left(\Delta_{E_{\mathrm{u}, 1}}, \Delta_{E_{\mathrm{u}, 2}}\right)\end{array}$ & $\begin{array}{l}1 \\
\left(\begin{array}{ll}1 & 0 \\
0 & 1\end{array}\right)\end{array}$ & $\begin{array}{l}1 \\
\left(\begin{array}{cc}-1 & 0 \\
0 & 1\end{array}\right)\end{array}$ & $\begin{array}{l}-1 \\
\left(\begin{array}{ll}0 & 1 \\
1 & 0\end{array}\right)\end{array}$ \\
\hline
\end{tabular}

Table 2. Point groups of the three solutions at $\Gamma$ and along $\Sigma_{x(y)}$ directions.

\begin{tabular}{llll}
\hline Solution & $\Gamma$ & $\Sigma_{x}$ & $\Sigma_{y}$ \\
\hline$a$ & $D_{4 h}$ & $C_{2 v}$ & $C_{2 v}$ \\
$\beta$ & $D_{2 h}$ & $C_{2 v}$ & $C_{2 v}$ \\
$\gamma$ & $C_{2 v}$ & $C_{2 v}$ & $C_{s}$ \\
\hline
\end{tabular}

population between the $d_{x z}, d_{y z}$ and $d_{x y}$ orbitals below $T_{\mathrm{s}}$ was recently noticed in experiment ${ }^{52}$.

Inversion symmetry breaking. Another important difference between the $C_{2 v}$ and the $D_{2 h}$ point group is that $C_{2 v}$ does not contain inversion symmetry. Direct measurement sensitive to electronic inversion symmetry breaking is now possible, thanks to the second harmonic generation (SHG) technique ${ }^{53-55}$. The prediction is that if the $\Delta_{E_{\mathrm{u}, 1(2)}}$ OP occurs, an unambiguous SHG signal should be observed below $T_{\mathrm{s}} . \Delta_{E_{\mathrm{u} 1(2)}}$ is conjugate to an inplane electric field $\mathbf{E}$ along one of the $\mathrm{Fe}-\mathrm{Fe}$ bonding directions. This will lead to a Dresselhaus splitting $\sim(\mathbf{E} \times \mathbf{k}) \cdot \mathbf{S}$, in which $\mathbf{S}$ is the electron spin operator. The splitting is largest along the $\mathbf{k}$ direction perpendicular to $\mathbf{E}$, and vanishes when $\mathbf{k} \| \mathbf{E}$. This term also leads to an out-of-plane polarization of the Fe spins, which is consistent with the spin-polarized inelastic neutron scattering data $^{16}$. We quantify the Dresselhaus splitting by acting spin-orbit coupling (SOC) upon the PM $\gamma$ solution as a first-order perturbation. Due to the overestimated band width, the SOC effect can be barely observed in Fig. 7(a), but a zoomed-in view [Fig. 7(b)] clearly shows the directional splitting of the order of $O(10) \mathrm{meV}$. This is also considered as a signature of $\Delta_{E_{u}}$, which can in principle be resolved in high-precision ARPES data, e.g., ref. ${ }^{56}$, and the Supplementary material of ref. ${ }^{34}$. In the same way as $\Delta_{B_{19}}$ couples to a shear strain of the lattice ${ }^{7}, \Delta_{E_{u}}$ couples to a special type of structural distortion. According to the full relaxation at the hybrid functional level, a relative glide between the $\mathrm{Fe}$ and Se layers occurs along one of the Fe-Fe bonding directions, which also breaks the inversion symmetry in analogy to the polar distortion in ferroelectric materials. However, the calculated magnitude is as small as $0.001 \AA$, which again reflects that lattice instability is not the driving force.

Pressure effect. The band evolution under reduced in-plane lattice constant and the out-of-plane distance between the $\mathrm{Fe}$ and Se layers (Fig. 6b, c) can be regarded as an examination of the pressure effect. Our results are consistent with the experimental 

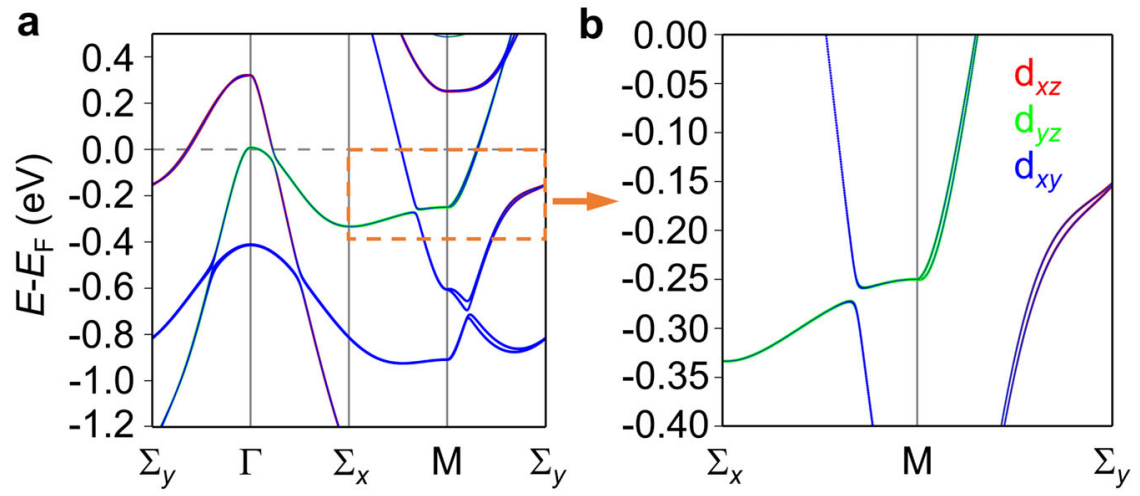

Fig. 7 The effect of SOC. a Ground-state ( $\gamma$ solution) band structure including SOC; $\mathbf{b}$ directional Dresselhaus splitting around the electron pocket.

observation of a strong reduction of the nematic transition temperature under pressure ${ }^{20}$. According to the experimental phase diagram ${ }^{20}$, while the nematic phase is suppressed, the stripe antiferromagnetic phase emerges. This instability is not covered in the present nonmagnetic framework. It is believed that a quantum phase transition occurs along the pressure axis. Beyond a critical point, the PM condition no longer applies.

\section{Perspective}

In summary, we demonstrated a first-principle approach to reproduce the PM nematic state in FeSe, without breaking either the tetragonal lattice symmetry or the time-reversal symmetry. We incorporated orbital-resolved interactions by $+U$ and hybrid functional, and preconditioned the initial wavefunction to find self-consistent solutions with spontaneous symmetry breaking. The lowest-energy nematic state we found features a twocomponent vector $\mathrm{OP}$ belonging to the $E_{\mathrm{u}}$ irrep, in addition to the Ising ferro-orbital order, which is important to produce the correct Fermi surface topology. We proposed that the inversion symmetry breaking induced by the $E_{\mathrm{u}}$ OP could be detected by high-precision measurement of the band dispersion as well as SHG. For the closely related material FeTe, a SHG experiment has already been reported, observing anomalies across the symmetrybreaking transition ${ }^{55}$.

From the computational side, it is worth further investigation to generalize the present nematic solutions to more advanced firstprinciple methods with interaction corrections beyond the Hartree-Fock level. If one views the $+U$ correction as the simplest DMFT with a static onsite self energy ${ }^{57}$, it is reasonable to expect that a more accurate treatment of the onsite correlation via a quantum impurity solver should better reproduce the experiment. Technically, the main issue is how to precondition the initial Green's function. If the conventional construction out of the converged LDA (GGA) Hamiltonian cannot automatically guide the DFT-DMFT loops to the nematic solutions, some alternative starting points have to be chosen. Similarly, if one views the GW method as a dynamical hybrid functional ${ }^{58}$, it is reasonable to expect that DFT $+\mathrm{GW}$ is also sufficient to reproduce nematicity in FeSe.

\section{METHODS}

Rountine setup

All the presented calculation results are obtained by using the Vienna ab initio Simulation Package (VASP $)^{59}$. The lattice structure respects the full $P 4 / n m m$ space group symmetry throughout the DFT $+U$ study. The experimental lattice parameters of bulk $\mathrm{FeSe}^{60}$ are used as the reference to obtain the main results. As a benchmark, we also perform full structural relaxation using the HSE06 ${ }^{61,62}$ hybrid functional. The atomic positions are relaxed until the residual forces are smaller than $5 \times 10^{-3} \mathrm{eV} \AA^{-1}$. In the end, the in-plane lattice constant and the Se height are manually varied separately to understand their effects.

The plane-wave cutoff is $500 \mathrm{eV}$ in combination with the projector augmented wave method ${ }^{63}$. The Monkhorst-Pack ${ }^{64}$ k-point grid is $12 \times$ $12 \times 6$. The exchange and correlation is treated by using the Perdew-Burke-Ernzerhof GGA functional ${ }^{65}$. The convergence criteria is $10^{-6} \mathrm{eV}$ for electronic iterations. The automatic symmetrization routine is switched off to probe electronic spontaneous symmetry breaking.

Unless specified otherwise, the presented calculation results include the rotational invariant $+U$ correction introduced by Dudarev et al. ${ }^{66}$ with $U=$ $3.6 \mathrm{eV}$ (or more rigorously, $U-J=3.6 \mathrm{eV}$ ). The more complicated orbitaldependent $+U$ correction as introduced by Liechtenstein et al. ${ }^{67}$ with $U=$ $F_{0}=4.8 \mathrm{eV}$ and $J=\left(F_{2}+F_{4}\right) / 14=1.2 \mathrm{eV}$ and $F_{2} / F_{4}=0.625$ gives very similar results. This $+U$ parameter is selected by benchmarking the band structure to HSE06 hybrid functional results, which contains no materialspecific parameter. In the end, we purposely tune Dudarev's $+U$ parameter from 3.2 to $4.0 \mathrm{eV}$ to understand its effect on the results.

\section{Wavefunction preconditioning}

To target the lowest-energy solution, various initial wavefunctions for the DFT iteration are tested. We first generate a set of $\psi_{i}(\mathbf{r})$ from a preparatory calculation on a manually distorted FeSe lattice that slightly breaks the symmetry. Then this set of $\left\{\psi_{i}(\mathbf{r})\right\}$ is fed to an undistorted FeSe lattice as a starting point to see whether it flows to a different local minimum. No matter how the preconditioning is performed, the energy landscape for the production run does not change, and is always ensured to be an invariant of the space group $P 4 / \mathrm{nmm}$. Specifically, we find that preconditioned wavefunctions generated by an uniaxial strain along the $x$ (or equally $y$ ) axis (see the inset of Fig. 1 for the definition of the $x$-axis) tend to flow into the $\beta$ basin. For the $y$ solution, the most convenient preconditioning is to shift the origin of the Fe layer along either the $x$ or the $y$ axis. We have tested wavefunction preconditioning associated with all the zone-center optical phonon modes (with VASP), as well as imposing the initial density matrix of the Fe $3 d$-orbitals (with the ABINIT code ${ }^{50,68,69}$ ) to cover all the ten irreps of the $D_{4 \mathrm{~h}}$ point group, which confirm that the $\gamma$ solution has the lowest energy. Certain minimization algorithms ${ }^{70}$ are able to escape shallow local minima. We find that without preconditioning, the damped velocity friction algorithm (See for example Sec. III of ${ }^{70}$ ) can still correctly find the $y$ solution as the ground state, despite a significantly larger number of iterations.

\section{Validity and limitations}

For the electronic structure above the nematic transition temperature $\left(T_{\mathrm{s}} \sim\right.$ $90 \mathrm{~K}$ for FeSe), LDA (GGA) instead of LSDA (sGGA) is the common choice to construct the tight-binding model (See, for example, Chpt. 8 in ref. ${ }^{1}$ ). Across $T_{s}$, the PM condition of the metallic state does not significantly change $e^{19,71}$. Despite an oversimplified description of paramagnetism, we consider that the symmetric LDA (GGA) band structure is eligible to serve as the numerical parent state, upon which we test whether the residual interaction effects drive any orbital instability at the Hartree-Fock level.

The main limitation of our calculation is the missing of dynamical spin fluctuation and short-range antiferromagnetic correlation. A traditional 
DFT approach to describe high-temperature PM states is to perform random average of different magnetically ordered states under LSDA (sGGA). However, the low-temperature quantum paramagnetism in FeSe has a much more complicated nature. Without an accurate knowledge of the spin ground state, the average is problematic. A perspective on generalizing the present calculation to more advanced algorithms is discussed in the end of the main context.

\section{DATA AVAILABILITY}

The data that support the findings of this study are available from the corresponding author upon reasonable request.

Received: 25 February 2020; Accepted: 3 July 2020; Published online: 24 July 2020

\section{REFERENCES}

1. Wang, N. L., Hosono, H. \& Dai, P. Iron-based Superconductors: Materials, Properties and Mechanisms (CRC Press, 2012).

2. Johnson, P. D., Xu, G. \& Yin, W.-G. Iron-based Superconductivity, vol. 211 (Springer, 2015).

3. Richard, P., Sato, T., Nakayama, K., Takahashi, T. \& Ding, H. Fe-based superconductors: an angle-resolved photoemission spectroscopy perspective. Rep. Prog. Phys. 74, 124512 (2011).

4. Dai, P., Hu, J. \& Dagotto, E. Magnetism and its microscopic origin in iron-based high-temperature superconductors. Nat. Phys. 8, 709-718 (2012).

5. Yin, Z., Haule, K. \& Kotliar, G. Kinetic frustration and the nature of the magnetic and paramagnetic states in iron pnictides and iron chalcogenides. Nat. Mater. 10, 932-935 (2011).

6. Yin, Z., Haule, K. \& Kotliar, G. Magnetism and charge dynamics in iron pnictides. Nat. Phys. 7, 294-297 (2011).

7. Fernandes, R. M., Chubukov, A. V. \& Schmalian, J. What drives nematic order in iron-based superconductors? Nat. Phys. 10, 97-104 (2014).

8. Fedorov, A. et al. Effect of nematic ordering on electronic structure of FeSe. Sci. Rep. 6, 36834 (2016).

9. Chu, J.-H., Kuo, H.-H., Analytis, J. G. \& Fisher, I. R. Divergent nematic susceptibility in an iron arsenide superconductor. Science 337, 710-712 (2012).

10. Fang, C., Yao, H., Tsai, W.-F., Hu, J. \& Kivelson, S. A. Theory of electron nematic order in LaFeAsO. Phys. Rev. B 77, 224509 (2008).

11. Xu, C., Müller, M. \& Sachdev, S. Ising and spin orders in the iron-based superconductors. Phys. Rev. B 78, 020501 (R) (2008).

12. Fernandes, R., Chubukov, A., Knolle, J., Eremin, I. \& Schmalian, J. Preemptive nematic order, pseudogap, and orbital order in the iron pnictides. Phys. Rev. B 85, 024534 (2012)

13. Lee, C.-C., Yin, W.-G. \& Ku, W. Ferro-orbital order and strong magnetic anisotropy in the parent compounds of iron-pnictide superconductors. Phys. Rev. Lett. 103, 267001 (2009).

14. Chen, C.-C. et al. Orbital order and spontaneous orthorhombicity in iron pnictides. Phys. Rev. B 82, 100504(R) (2010).

15. Kontani, H., Saito, T. \& Onari, S. Origin of orthorhombic transition, magnetic transition, and shear-modulus softening in iron pnictide superconductors: analysis based on the orbital fluctuations theory. Phys. Rev. B 84, 024528 (2011).

16. Ma, M. et al. Prominent role of spin-orbit coupling in FeSe revealed by inelastic neutron scattering. Phys. Rev. X 7, 021025 (2017).

17. Anisimov, V. I., Aryasetiawan, F. \& Lichtenstein, A. First-principles calculations of the electronic structure and spectra of strongly correlated systems: the LDA $+U$ method. J. Phys. Condens. Matter 9, 767-808 (1997).

18. Becke, A. D. A new mixing of Hartree-Fock and local density-functional theories. J. Chem. Phys. 98, 1372-1377 (1993).

19. Bendele, M. et al. Coexistence of superconductivity and magnetism in $\mathrm{FeSe}_{1-x}$ under pressure. Phys. Rev. B 85, 064517 (2012).

20. Sun, J. et al. Dome-shaped magnetic order competing with high-temperature superconductivity at high pressures in FeSe. Nat. Commun. 7, 12146 (2016).

21. Qing-Yan, W. et al. Interface-induced high-temperature superconductivity in single unit-cell FeSe films on $\mathrm{SrTiO}_{3}$. Chin. Phys. Lett. 29, 037402 (2012).

22. $\mathrm{Ge}, \mathrm{J} .-\mathrm{F}$. et al. Superconductivity above $100 \mathrm{~K}$ in single-layer FeSe films on doped $\mathrm{SrTiO}_{3}$. Nat. Mater. 14, 285-289 (2015).

23. Nakayama, K. et al. Reconstruction of band structure induced by electronic nematicity in an FeSe superconductor. Phys. Rev. Lett. 113, 237001 (2014).

24. Shimojima, T. et al. Lifting of $x z / y z$ orbital degeneracy at the structural transition in detwinned FeSe. Phys. Rev. B 90, 121111(R) (2014).
25. Suzuki, Y. et al. Momentum-dependent sign inversion of orbital order in superconducting FeSe. Phys. Rev. B 92, 205117 (2015).

26. Zhang, P. et al. Observation of two distinct $d_{x z} / d_{y z}$ band splittings in FeSe. Phys. Rev. B 91, 214503 (2015).

27. Watson, M. et al. Emergence of the nematic electronic state in FeSe. Phys. Rev. B 91, 155106 (2015).

28. Watson, M. et al. Evidence for unidirectional nematic bond ordering in FeSe. Phys. Rev. B 94, 201107(R) (2016).

29. Watson, M. D., Haghighirad, A. A., Rhodes, L. C., Hoesch, M. \& Kim, T. K. Electronic anisotropies revealed by detwinned angle-resolved photo-emission spectroscopy measurements of FeSe. New J. Phys. 19, 103021 (2017).

30. Rhodes, L. C. et al. Scaling of the superconducting gap with orbital character in FeSe. Phys. Rev. B 98, 180503(R) (2018).

31. $\mathrm{Xu}, \mathrm{H}$. et al. Highly anisotropic and twofold symmetric superconducting gap in nematically ordered $\mathrm{FeSe}_{0.93} \mathrm{~S}_{0.07}$. Phys. Rev. Lett. 117, 157003 (2016).

32. Zhang, Y. et al. Distinctive orbital anisotropy observed in the nematic state of a FeSe thin film. Phys. Rev. B 94, 115153 (2016).

33. Fanfarillo, L. et al. Orbital-dependent Fermi surface shrinking as a fingerprint of nematicity in FeSe. Phys. Rev. B 94, 155138 (2016).

34. Kushnirenko, Y. et al. Three-dimensional superconducting gap in FeSe from angle-resolved photoemission spectroscopy. Phys. Rev. B 97, 180501(R) (2018).

35. Liu, D. et al. Orbital origin of extremely anisotropic superconducting gap in nematic phase of FeSe superconductor. Phys. Rev. X 8, 031033 (2018).

36. Wang, Z. et al. Topological edge states in a high-temperature superconductor FeSe/SrTiO 3 (001) film. Nat. Mater. 15, 968-973 (2016).

37. $\mathrm{Yi}, \mathrm{M}$. et al. Nematic energy scale and the missing electron pocket in FeSe. Phys. Rev. X 9, 041049 (2019).

38. Huh, S. et al. Absence of Y-pocket in 1-Fe Brillouin zone and reversed orbital occupation imbalance in FeSe. Commun. Phys. 3, 52 (2020).

39. Su, Y., Liao, H. \& Li, T. The form and origin of orbital ordering in the electronic nematic phase of iron-based superconductors. J. Phys. Condens. Matter 27, 105702 (2015).

40. Onari, S., Yamakawa, Y. \& Kontani, H. Sign-reversing orbital polarization in the nematic phase of FeSe due to the $C_{2}$ symmetry breaking in the self-energy. Phys. Rev. Lett. 116, 227001 (2016).

41. Xing, R.-Q., Classen, L. \& Chubukov, A. V. Orbital order in FeSe: the case for vertex renormalization. Phys. Rev. B 98, 041108(R) (2018).

42. Yi, M., Zhang, Y., Shen, Z.-X. \& Lu, D. Role of the orbital degree of freedom in ironbased superconductors. npj Quant. Mater. 2, 57 (2017).

43. Sprau, P. O. et al. Discovery of orbital-selective Cooper pairing in FeSe. Science 357, 75-80 (2017).

44. Kostin, A. et al. Imaging orbital-selective quasiparticles in the Hund's metal state of FeSe. Nat. Mater. 17, 869-874 (2018).

45. Glasbrenner, J. et al. Effect of magnetic frustration on nematicity and superconductivity in iron chalcogenides. Nat. Phys. 11, 953-958 (2015).

46. Liu, K., Lu, Z.-Y. \& Xiang, T. Nematic antiferromagnetic states in bulk FeSe. Phys. Rev. B 93, 205154 (2016).

47. Wang, F., Kivelson, S. A. \& Lee, D.-H. Nematicity and quantum paramagnetism in FeSe. Nat. Phys. 11, 959-963 (2015).

48. Cococcioni, M. \& De Gironcoli, S. Linear response approach to the calculation of the effective interaction parameters in the LDA $+U$ method. Phys. Rev. $B$ 71, 035105 (2005).

49. Meredig, B., Thompson, A., Hansen, H. A., Wolverton, C. \& van de Walle, A. Method for locating low-energy solutions within DFT+U. Phys. Rev. B 82, 195128 (2010).

50. Amadon, B., Jollet, F. \& Torrent, M. $\gamma$ and $\beta$ cerium: LDA $+U$ calculations of groundstate parameters. Phys. Rev. B 77, 155104 (2008).

51. Cvetkovic, V. \& Vafek, O. Space group symmetry, spin-orbit coupling, and the lowenergy effective hamiltonian for iron-based superconductors. Phys. Rev. B 88, 134510 (2013).

52. Li, J. et al. Spin-orbital-intertwined nematic state in FeSe. Phys. Rev. X 10, 011034 (2020).

53. Zhao, L. et al. A global inversion-symmetry-broken phase inside the pseudogap region of $\mathrm{YBa}_{2} \mathrm{Cu}_{3} \mathrm{O}_{y}$. Nat. Phys. 13, 250-254 (2017).

54. Harter, J., Chu, H., Jiang, S., Ni, N. \& Hsieh, D. Nonlinear and time-resolved optical study of the 112-type iron-based superconductor parent $\mathrm{Ca}_{1-x} \mathrm{La}_{x} \mathrm{FeAs}_{2}$ across its structural phase transition. Phys. Rev. B 93, 104506 (2016).

55. Kityk, I., Viennois, R. \& Plucinski, K. Phase transition diagnostic in iron telluride by nonlinear optical experiments. Acta Phys. Pol. A 121, 932-935 (2012).

56. $\mathrm{Li}, \mathrm{C}$. et al. Evidence for an additional symmetry breaking from direct observation of band splitting in the nematic state of FeSe superconductor. https://journals. aps.org/prx/accepted/ae073K31C6016107a4ba31f563009819a004318b2 (2019).

57. Kotliar, G. et al. Electronic structure calculations with dynamical mean-field theory. Rev. Mod. Phys. 78, 865-951 (2006).

58. Hedin, L. On correlation effects in electron spectroscopies and the GW approximation. J. Phys. Condens. Matter 11, R489-R528 (1999). 
59. Kresse, G. \& Furthmüller, J. Efficient iterative schemes for abinitio total-energy calculations using a plane-wave basis set. Phys. Rev. B 54, 11169-11186 (1996).

60. McQueen, T. M. et al. Extreme sensitivity of superconductivity to stoichiometry in $\mathrm{Fe}_{1+\delta}$ Se. Phys. Rev. B 79, 014522 (2009).

61. Heyd, J., Scuseria, G. E. \& Ernzerhof, M. Hybrid functionals based on a screened Coulomb potential. J. Chem. Phys. 118, 8207-8215 (2003).

62. Heyd, J., Scuseria, G. E. \& Ernzerhof, M. Erratum: "Hybrid functionals based on a screened Coulomb potential" [J. Chem. Phys. 118, 8207 (2003)]. J. Chem. Phys. 124, 219906 (2006)

63. Blöchl, P. E. Projector augmented-wave method. Phys. Rev. B 50, 17953-17979 (1994).

64. Monkhorst, H. J. \& Pack, J. D. Special points for Brillouin-zone integrations. Phys. Rev. B 13, 5188-5192 (1976).

65. Perdew, J. P., Burke, K. \& Ernzerhof, M. Generalized gradient approximation made simple. Phys. Rev. Lett. 77, 3865-3868 (1996).

66. Dudarev, S., Botton, G., Savrasov, S., Humphreys, C. \& Sutton, A. Electron-energyloss spectra and the structural stability of nickel oxide: an LSDA+U study. Phys. Rev. B 57, 1505-1509 (1998).

67. Liechtenstein, A., Anisimov, V. \& Zaanen, J. Density-functional theory and strong interactions: Orbital ordering in Mott-Hubbard insulators. Phys. Rev. B 52, R5467-R5470 (1995).

68. Gonze, X. et al. The ABINIT project: Impact, environment and recent developments. Comput. Phys. Commun. 248, 107042 (2020).

69. Torrent, M., Jollet, F., Bottin, F., Zérah, G. \& Gonze, X. Implementation of the projector augmented-wave method in the ABINIT code: application to the study of iron under pressure. Comput. Mater. Sci. 42, 337-351 (2008).

70. Payne, M. C., Teter, M. P., Allan, D. C., Arias, T. \& Joannopoulos, aJ. Iterative minimization techniques for abinitio total-energy calculations: molecular dynamics and conjugate gradients. Rev. Mod. Phys. 64, 1045-1097 (1992).

71. Wang, Q. et al. Magnetic ground state of FeSe. Nat. Commun. 7, 12182 (2016).

\section{ACKNOWLEDGEMENTS}

We would like to thank Ming Yi, Yan Zhang, Wei Li, Yuan Li, Tao Wu, Yi Zhou, Yuan Wan, Hong Yao, and Yuanming Lu for helpful discussion. This work is supported by NSFC under Grant no. 11774196 and Tsinghua University Initiative Scientific Research Program. F.W. acknowledges support from the National Key Research and Development Program of China (Grand no. 2017YFA0302904). S.Z. acknowledges support from NSFC (11904350) and Anhui Provincial Natural Science Foundation (2008085QA30).

\section{AUTHOR CONTRIBUTIONS}

X.L and Z.L. conceived the project. X.L. and S.Z. performed the calculations. F.W contributed to the theoretical interpretations. All the authors prepared the manuscript.

\section{COMPETING INTERESTS}

The authors declare no competing interests.

ADDITIONAL INFORMATION

Correspondence and requests for materials should be addressed to Z.L.

Reprints and permission information is available at http://www.nature.com/ reprints

Publisher's note Springer Nature remains neutral with regard to jurisdictional claims in published maps and institutional affiliations.

Open Access This article is licensed under a Creative Common Attribution 4.0 International License, which permits use, sharing, adaptation, distribution and reproduction in any medium or format, as long as you give appropriate credit to the original author(s) and the source, provide a link to the Creative Commons license, and indicate if changes were made. The images or other third party material in this article are included in the article's Creative Commons license, unless indicated otherwise in a credit line to the material. If material is not included in the article's Creative Commons license and your intended use is not permitted by statutory regulation or exceeds the permitted use, you will need to obtain permission directly from the copyright holder. To view a copy of this license, visit http://creativecommons. org/licenses/by/4.0/.

(c) The Author(s) 2020 